\title{
Some Environmental Factors Affecting the Length of Escherichia coli Organisms in Continuous Cultures
}

\author{
By F. I. MACLEAN AND R. J. MUNSON \\ Medical Research Council Radiobiological Research Unit, \\ Harwell, Didcot, Berkshire
}

(Received 26 August 1960)

\begin{abstract}
SUMMARY
The lengths of organisms of Escherichia coli $\mathrm{B} / \mathrm{r}$ grown in continuous culture in a glucose salts medium were measured over a wide range of population densities at $22^{\circ}$ and $37^{\circ}$. In some cases the cultures were exposed to continuous gamma radiation at dose rates of $600 \mathrm{r}$. $/ \mathrm{hr}$. at $22^{\circ}$ and of $1000 \mathrm{r}$. $/ \mathrm{hr}$. at $37^{\circ}$. The average length of the forms and the distribution with respect to length depended upon the temperature, population density and radiation dose rate. The growth rate was almost independent of population density over the range in which the average length of organism showed such a marked dependence. No significant differences in growth rate were observed as between unirradiated and irradiated cultures. Changes in average length of organisms are regarded as phenotypic responses to changes in growth conditions, some conditions favouring division and others inhibiting division amongst short organisms.
\end{abstract}

\section{INTRODUCTION}

One of the advantages of a continuous culture system for bacteria is that the organisms can be maintained in active division for many generations in an unchanging environment. Such a system is very suitable for the study of the production of spontaneous or induced mutants (Novick \& Szilard, 1950) and has been used in our experiments on the effects of continuous gamma irradiation on actively growing cultures of Escherichia coli. The effect with which we are here concerned is the appearance of long filamentous forms in the bacterial population in both irradiated and unirradiated cultures. At the outset it seemed likely that these long forms might have some properties different from those of the 'normal' shorter bacteria, and that their relative proportions would affect the behaviour of the culture as a whole. For example, the radio-resistance of the long forms might differ from that of the short ones (Lea, Haines \& Coulson, 1937) or the long ones might represent the emergence of a new strain (Hughes, 1953). Very long forms have been observed in cultures subjected to gamma radiation (Lea et al. 1937), $\mathrm{X}$ radiation (Alper, 1957), ultraviolet radiation (Witkin, 1947), sudden changes in osmotic pressure (Hinshelwood \& Lodge, 1944), antibiotics (Fleming, Voureka, Kramer \& Hughes, 1950) and growth inhibitors (Spray \& Lodge, 1943). In all these cases the additional factor superimposed on the environmental conditions was responsible for the inhibition of normal cell division. Some inhibition of division must also occur in cultures grown 
in broth in the usual way since large differences in length as between individual organisms are found. The average size of bacteria in batch cultures also depends upon the nutrient concentration and phase of growth, as shown by the systematic studies of Henrici (1928). Observations of long-organism formation in batch cultures cannot therefore be interpreted in a simple manner. The provision of an environment which remains unaltered over many generation times would appear to be a basal requirement for work in this and other fields where population changes occupy more than a very small number of generation times. A continuous culture system is admirably suited to this purpose.

Some observations on the relation between growth rate and bacterial size in continuous cultures have already been reported (Schaechter, Maaløe \& Kjeldgaard, 1958; Herbert, 1959). Our own observations show a close correlation between the average size of organism and the proportion of abnormally long forms, each being dependent upon the population density, the temperature and radiation dose rate.

\section{Growth rate and length distribution in continuous cultures}

When Escherichia coli organisms are examined under the microscope different forms can be seen which might be appropriately described as normal rods, as chains or as filaments. Such a classification has not been attempted in the present work, and as a consequence we have found the need for an inclusive term to cover these and other forms. For convenience we have adopted the word 'cell' to cover any of these various forms. We count as one 'cell' any bacterium which is recognizably separate from its neighbours in liquid suspension and moves amongst them as one entity, although in the process it may suffer changes in internal configuration by Brownian movement.

Suppose that at time $t$ the number of cells/unit volume of a continuous culture is $N$ and the dilution rate, i.e. the rate of flow divided by the capacity of the culture vessel, is $D$. If one assumes that the culture is always uniformly mixed then

$$
\frac{d N}{d t}=N(\bar{\nu}-D)
$$

where $\bar{\nu}$ is the apparent rate at which new organisms are 'born' or the number growth rate (Powell, 1956) for the whole population. If $\bar{\nu}$ is independent of $N$ and of $t$, and if $D$ can be regarded as constant during a time interval $t-t_{0}$, integration of (1) gives

$$
\ln \left(N / N_{0}\right)=(\bar{\nu}-D)\left(t-t_{0}\right),
$$

where $N_{0}$ is the population density at time $t_{0}$. Thus the graph of $\ln N$ against $t$ should have a constant slope, the value of which will give $\bar{\nu}$ when $D$ is known. In practice when $D$ is kept constant and $\bar{\nu}>D$ initially, $\bar{\nu}$ will eventually decrease as $N$ becomes large, due to exhaustion of the medium and other causes, but changes in $\vec{\nu}$ may also arise from changes in the average cell size or mass. For example, when the mass growth rate $\bar{\mu}$ of the whole culture (Powell, 1956) is constant and the average mass of the cells increases at the rate $\epsilon$ the number growth rate will be $\bar{\mu}-\epsilon$.

The average diameters of Escherichia coli organisms under given cultural conditions are almost independent of their length (Deering, 1958; this paper, page 25), so it will be assumed in the following analysis that the mass of a cell is proportional to its length. 
A continuous culture selects in favour of rapidly dividing cells from the time it is started, but several generation times may be required before the maximum and final growth rate is reached. During this early phase the distribution of generation times amongst the population changes (Powell, 1956), and consequently the distribution with respect to length usually changes also. Both distributions eventually become stable when the population density, temperature and other factors are kept constant and no further selection occurs. Such a culture will be called a steady-state culture, although the term has meaning only in relation to the property which is stable. In our case this is the length distribution, for which an infinite number of steady states seems possible, at least in theory.

The form of the length distribution in a bacterial population is determined by the time dependence of the processes of growth and division of the individual cells. Apart from studies of the distribution of generation times amongst a population (Powell, 1955, 1958) and some isolated observations of exponential growth of cells in which division had been inhibited by radiation (Lea et al. 1937; Deering, 1958) little is known about these processes in quantitative terms. It is possible that some information in this direction could be gained by constructing hypothetical models of the processes and comparing the length distributions predicted from them with those observed, but this will not be attempted here. Instead, we shall assume a simple exponential growth function and apply it to two special cases which represent extreme or limiting cases of the more general one.

In an unchanging environment the mass growth rate $\bar{\mu}$ of a population is constant so we shall assume, as a first approximation, that the growth rate of each cell is $\bar{\mu}$, i.e. each cell increases in length by the factor $e^{-\bar{\mu} t}$ in time $t$.

(1) In the first case suppose that all cells divide at a certain length $2 l_{1}$, and that all daughter cells have an initial length $l_{1}$. Cells of length $l_{1}$ at time zero will have lengths given by

$$
l=l_{1} e^{\bar{\mu} d t}
$$

at time $d t$. If at time zero there are $n\left(l_{1}\right) d l$ cells with lengths between $l_{1}$ and $l_{1}+d l$ a fraction $l_{1} / l$ of this number will have lengths between $l$ and $l+d l$ after time $d t$ provided they have not been washed away in the interval $d t$. Since the fraction surviving wash-out in this interval is $e^{-D d t}\left(=e^{-\bar{\mu} d t}\right.$, since $D=\bar{\mu}$ in a steady-state culture) and the number of cells in any length range does not change with time, the number between $l$ and $l+d l$ will be

$$
n(l)=n\left(l_{1}\right)\left(l_{1} / l\right) e^{-\bar{\mu} d t} d l,
$$

which by (3) can be written

$$
n(l)=n\left(l_{1}\right)\left(l_{1} / l\right)^{2} d l .
$$

When $n(l)$ is plotted against $l / l_{1}$ using double logarithmic scales as in Fig. $7 a$ the graph has a slope -2 for $2 \geqslant l / l_{1} \geqslant 1$. Outside this length range $n(l)$ is zero. In any real culture all cells would not divide at precisely the same length nor would they divide to give two exactly equal daughters. The ends of the distribution would therefore not be sharp but rounded as in the experimental curve to which Fig. $7 a$ has been fitted.

(2) In the second case suppose that cells do not divide at length $2 l_{1}$, but continue to grow longer at the same rate $\bar{\mu}$ until they reach a length $q l_{1}$, where $q$ is a large 
positive integer. If they then divided into separate cells of length $l_{1}$, a steady-state culture would be possible and the length distribution for $q \geqslant l / l_{1} \geqslant 1$ would have a slope of -2 . This distribution is represented graphically by the line $(b)$ of Fig. 7 for which $q$ is assumed to be very large.

In passing, it may be noted that if divisions did not occur at one particular length but took place at all lengths greater than $2 l_{1}$, with similar frequencies, the average cell length and the slope of the length distribution would have values between those for the two extreme cases (1) and (2). Thus the average length $l_{d}$ at which cells pass through their first division would be correlated with the average length of the whole population and with the slope of their length distribution.

So far we have considered only steady-state cultures of cells behaving according to our simplified model. In practice it may not be possible to maintain the dilution rate at its steady-state value $D=\bar{\mu}=\bar{\nu}$ and it is therefore important to know whether short-term fluctuations in dilution rate are likely to influence the length distribution substantially. Let us suppose that a culture has been operated in a steady state with a dilution rate $D_{1}$ and that the dilution rate is suddenly increased to $D_{2}$. If $l_{d}$ is independent of dilution rate, all cells will have the same decreased chance of surviving wash-out after time $t$, viz. $e^{\left(D_{1}-D_{2}\right) t}$. The change in dilution rate per se would therefore leave the length distribution unchanged. However, if the product $\left(D_{1}-D_{2}\right) t$ were sufficiently large, the cell density would change appreciably and a dependence of $l_{d}$ upon cell density would result in an altered length distribution.

\section{METHODS}

In the experiments described below Escherichia coli $\mathrm{B} / \mathrm{r}$ derived from a stock kindly supplied by Miss T. Alper was grown in a glucose salts medium (Lederberg, 1950) at $\mathrm{pH} \mathrm{7.0} \mathrm{in} \mathrm{a} \mathrm{continuous} \mathrm{culture} \mathrm{system.} \mathrm{Starter} \mathrm{cultures} \mathrm{were} \mathrm{routinely}$ grown overnight at $37^{\circ}$ in minimal medium and samples of such cultures were used as inocula for continuous cultures. The culture vessels had capacities of approximately $9 \mathrm{ml}$. and each was supplied with air at the rate of $700 \mathrm{ml} . / \mathrm{hr} .^{-1}$ and with nutrient at a rate which could be adjusted by manual control of the hydrostatic head $h$ (Fig. 1). The positions of the reservoir $R$ and mixer $M$ having been fixed the dilution rate fell by $1-3 \%$ over $24 \mathrm{hr}$., depending upon the actual dilution rate, the area of free liquid surface in the reservoir and the head $h$. Normally the system was adjusted to give unrestricted growth at constant or nearly constant population density. The apparatus in the form here described therefore functioned as a crude turbidostat (Bryson, 1959). A stirrer consisting of a permanent magnet encased in stainless steel driven at $300 \mathrm{rev} . / \mathrm{min}$. provided efficient mixing of medium and air. With its free-running tyre at one end and a fixed tyre at the other, the stirrer was subject to a frictional turning couple set up by its forced revolution and this caused it to rotate about its axis. This form of stirrer always remained free from adherent bacterial growth even after running continuously for as long as 2 weeks. Gamma radiation was provided by a ${ }^{60} \mathrm{Co}$ source of 8 curies kindly placed at our disposal by Dr G. J. Neary.

During an experiment samples were withdrawn from the culture vessels from time to time by means of Pasteur pipettes. These samples (vol. $10^{-2} \mathrm{ml}$.) were examined microscopically, with dark ground illumination and a Thoma Hawksley counting 
chamber $25 \mu$ deep. It was customary to record the total count (about 200) and also the numbers of 'long cells', i.e. cells longer than $8 \mu$, seen over the whole or part of the ruled area. When the complete length distribution of a sample was required the lengths of all cells (about 200) within a given area were measured.

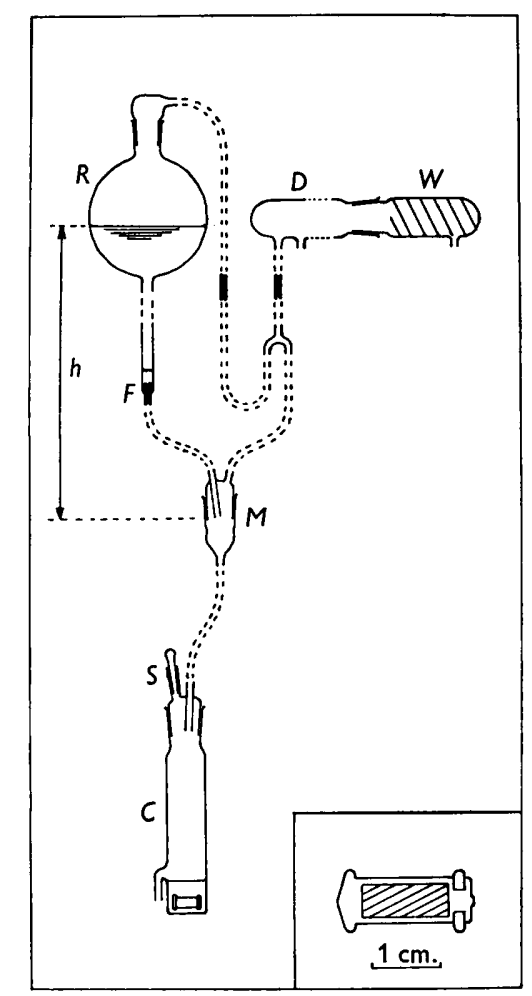

Fig. 1. Schematic diagram of the apparatus. Silicone rubber connexions between the glass components are shown by dashed lines. Heavy black lines indicate narrow tubes with appreciable flow resistance. $C=$ culture vessel; $S=$ sampling port; $M=$ mixer; $D=$ distributor of sterile humid air; $W=$ cotton wool plug; $F=$ filter; $R=$ reservoir of medium. Inset, magnetic stirrer.

\section{RESULTS}

The results to be described relate to experiments in which two or three cultures were inoculated from the same starter culture and run simultaneously, usually at different population densities. The dilution rate of each culture was adjusted so that it was about equal to the growth rate. During the first few hours changes were observed in \% long cells in the population and in the average cell length, but after several generation times both quantities reached apparently stable values. Figure 2 shows the changes in \% long cells in typical cultures during this initial period. After the $\%$ long cells had become constant observations were continued during several generation times (usually at least 10) so that sufficient data relating to the length distribution of cells in the chosen steady state could be obtained. In the following paragraphs the results given are for cultures which had reached, or appeared to have reached, stable length distributions. However it must be emphasized that the 
experiments were rarely continued for more than $24 \mathrm{hr}$. and therefore we have no evidence that the same effects would be found with much older cultures. The experimental errors in \% long-cell values were also relatively large so that over the short periods covered by the observations it was not possible to determine whether there was a tendency for the \% long cells to increase or decrease slowly.

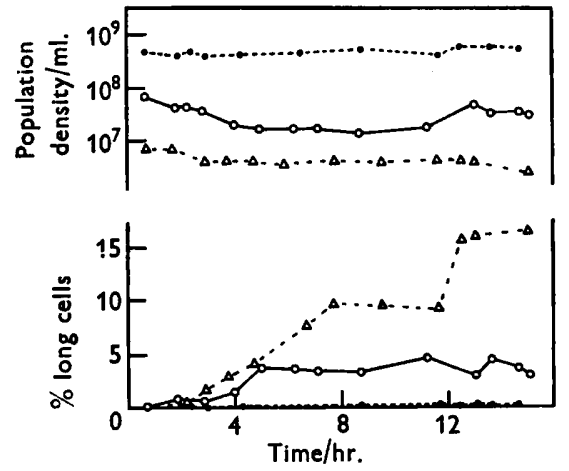

Fig. 2

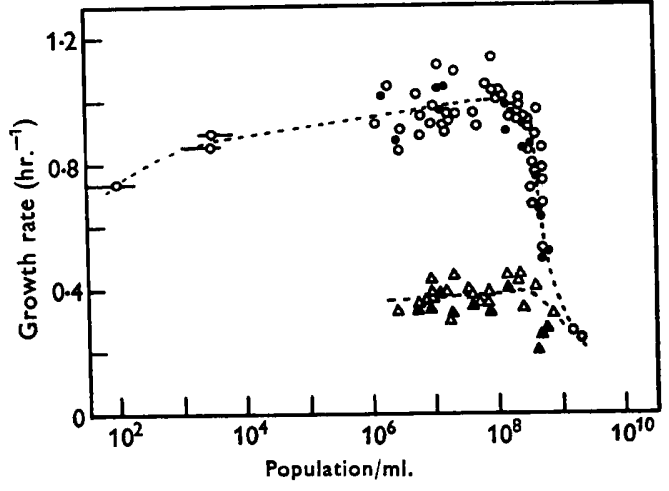

Fig. 3

Fig. 2. Changes in the proportion of cells which are long, i.e. greater than $8 \mu$ during the first few hours in three continuous cultures inoculated simultaneously from the same starter culture and run at $37^{\circ}$ at different population densities, namely: (O) $4 \times 10^{8} / \mathrm{ml}$; (O) $3 \times 10^{7} / \mathrm{ml}$; ( $\left.\triangle\right) 4 \times 10^{6} / \mathrm{ml}$.

Fig. 3. The growth rates of cultures as functions of population density. The extent of the large variation in population density for the three most dilute cultures is indicated by horizontal lines. Unirradiated cultures at $22^{\circ}(\triangle)$ and $37^{\circ}(O)$; irradiated cultures at 600 r. $/ \mathrm{hr}$. at $22^{\circ},(\Delta)$ and at 1000 r. $/ \mathrm{hr}$. at $37^{\circ}(\mathrm{O})$.

\section{Growth rates}

The number growth rate, which becomes equal to the mass growth rate in a steady-state culture, was calculated from experimental data by using equation (2). All values of growth rate for cultures grown at 22 and $37^{\circ}$, with and without gamma irradiation, are plotted in Fig. 3. It was estimated that they were subject to errors of about $5 \%$, due mainly to temperature fluctuations and to errors in the measurements of culture vessel volumes. Although there were wide differences between the results from experiment to experiment, a definite progressive decrease in growth rate at $37^{\circ}$ was evident at population densities greater than $2 \times 10^{8} / \mathrm{ml}$. due presumably to partial anoxia or nutrient limitation. A similar trend appeared at $22^{\circ}$ at a rather higher population density. At $\mathbf{3} 7^{\circ}$ observations were extended to population densities of about $10^{4}$ to $10^{2}$ cells $/ \mathrm{ml}$. by making viable counts (colonies at $72 \mathrm{hr}$. at $37^{\circ}$ on salts glucose agar) instead of the usual total counts.

Although growth rates for irradiated cultures were somewhat smaller on the average than those for unirradiated cultures at the same population density, the differences are not statistically significant.

\section{Proportion of long cells amongst population}

Figure 4 shows that there was an almost unique relation between the average cell length and \% long cells, particularly for unirradiated cultures. Since both of these 
quantities are dependent upon the length distribution, it follows that either could be used to specify such a distribution.

At $37^{\circ}$ the $\%$ long cells decreased towards zero as the cell concentration (population density) increased in the observed range, namely $10^{6}$ to $10^{9} / \mathrm{ml}$. This trend could be readily demonstrated in a single experiment such as the one represented in Fig. 2 in which three cultures from the same inoculum were run simultaneously at different population densities. However, when the results from many experiments were pooled in one diagram (as in Fig. 5), it became clear that there was not a unique relation between $\%$ long cells and population density.

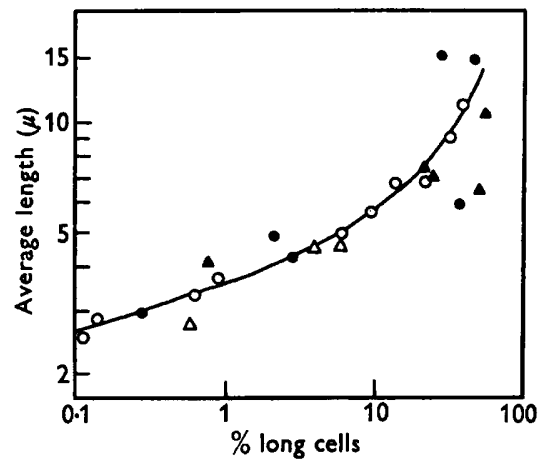

Fig. 4

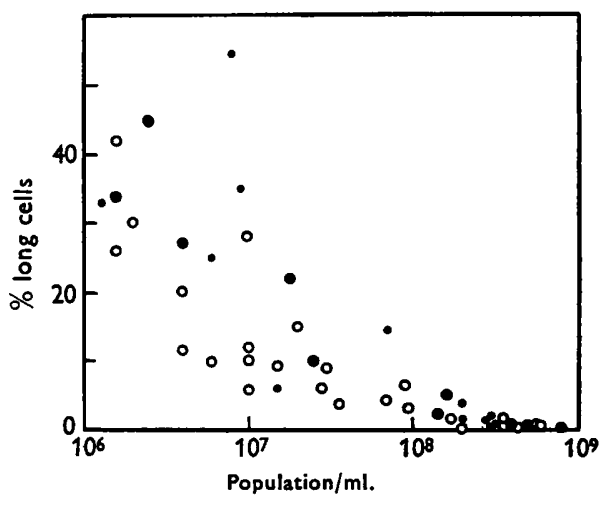

Fig. 5

Fig. 4. The average length of cells in a culture as a function of the $\%$ long cells. Points for irradiated and unirradiated cultures show no systematic differences. Symbols as Fig. 3.

Fig. 5. Observations from a large number of cultures showing the general form of dependence of $\%$ long cells upon population density at $37^{\circ}$. Points corresponding to cultures grown under continuous gamma irradiation at $1000 \mathrm{r} . / \mathrm{hr}$. (๑), and $600 \mathrm{r.} / \mathrm{hr}$. (•) tend to lie above those for cultures grown in the absence of radiation (O), but the differences are not statistically significant.

The results of Figs. 3 and 5 taken together show that the greatest changes in $\%$ long cells occurred in the range of population densities below $2 \times 10^{8} / \mathrm{ml}$. where the growth rate was almost constant. The average cell length in this population density range was therefore independent of the growth rate.

The effect of gamma radiation at dose rates up to $1000 \mathrm{r}$. $/ \mathrm{hr}$. at $37^{\circ}$ was tested by irradiating cultures after they had each reached an approximately steady state. The observed changes in the proportion of long cells were relatively small, and taken together did not give unequivocal evidence of an increase due to radiation at these dosages. The actual results are plotted in Fig. 5. Experiments were attempted at $43^{\circ}$, which is above the optimum temperature for growth, but reproducible results were not obtained.

The values of $\%$ long cells in unirradiated cultures at $22^{\circ}$ were only about onetenth of those values at $37^{\circ}$ with the same population density. For this reason and also because fewer observations were made, the evidence for a dependence of $\%$ long cells on population density at $22^{\circ}$, similar to that of Fig. 3, was not conclusive. However, the presence of such a small proportion of long cells in an unirradiated 
culture made the detection of a small increase due to gamma radiation correspondingly easier. The results of an experiment with cultures at two different population densities exposed to gamma radiation (600 r./hr.) are shown in Fig. 6. The increase in $\%$ long cells following irradiation was more marked at the smaller population density, the \% long cells reaching a value typical of those for unirradiated cultures at $37^{\circ}$. Thus an increase in temperature and exposure to gamma rays produced similar changes in the proportion of long cells.



Fig. 6

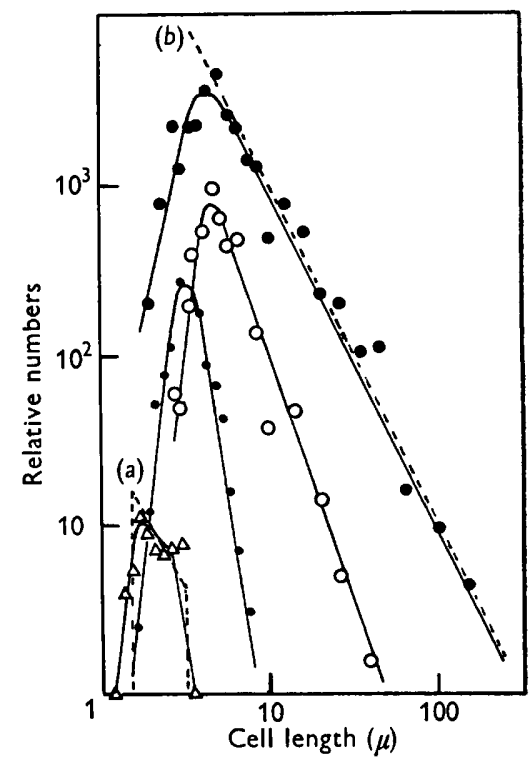

Fig. 7

Fig. 6. The effect of gamma irradiation at $600 \mathrm{r} . / \mathrm{hr}$. on $\%$ long cells in cultures at $22^{\circ}$, at high $\left(3 \times 10^{8} / \mathrm{ml}\right.$, O) and low $\left(3 \times 10^{7} / \mathrm{ml}\right.$., O) population densities. Irradiation commenced at $20 \cdot 6 \mathrm{hr}$.

Fig. 7. The number of cells observed/unit interval of length plotted against length for each of four cultures at $37^{\circ}$. The population densities were $10^{9} / \mathrm{ml} .(\Delta), 4 \times 10^{8} / \mathrm{ml}$. (•), $1.0 \times 10^{7} / \mathrm{ml}$. (O) in the absence of gamma radiation, and $1.2 \times 10^{7} / \mathrm{ml}$. (O) at $1000 \mathrm{r} . / \mathrm{hr}$. gamma radiation. The points at longer cell lengths represent average values of the frequency over several adjacent unit length intervals. The graph $(a)$ showing the distribution to be expected on the simplest assumptions (see text) has been fitted to one set of experimental points. The line $(b)$ shows the expected distribution for a population in which cells only divide when they are very long.

The general form of the length distribution of cells of Escherichia coli strain $\mathbf{B} / \mathbf{r}$ in continuous cultures is shown in Fig. 7. The number of cells with lengths between $l$ and $l+d l$ divided by $d l$, which may be denoted by $n(l)$, is plotted against $l$, using double logarithmic scales. At lengths greater than the mode, the distribution curves became linear within the limits of experimental error. An analysis of all the data showed that the slope of the linear portion and the average cell length were correlated (coefficient $0.76, P<0.001$ ) despite the wide range of conditions under which the cells were cultivated. It follows that the shapes of all distribution curves could be expressed in terms of the average length only, a conclusion independently 
confirmed by the almost unique relation existing between the average length and $\%$ long cells (Fig. 4). The uppermost curve of Fig. 7 for a culture at $37^{\circ}$ and irradiated at $1000 \mathrm{r} . / \mathrm{hr}$. is noteworthy in that the slope of its linear portion approximates to $\mathbf{- 2 \cdot 0}$. This, on our simple model, is the smallest possible value of the average slope for a culture in a steady state and obtains when cells which have reached a certain length grow longer without further division.

\section{Stability of cultures}

Since a change in population density generally caused a change in length distribution, a culture could only become strictly stable when the dilution rate was maintained exactly equal to the growth rate. In these experiments this condition was only fulfilled at population densities greater than $2 \times 10^{8} / \mathrm{ml}$. when the system behaved as a chemostat (Bryson, 1959) with the suboptimal growth rate (compare Fig. 2) adjusting itself to equality with the dilution rate. At population densities less than $2 \times 10^{8} / \mathrm{ml}$. any error in the adjustment of the dilution rate caused a steady increase or decrease in population density (see Equation 2). Over the period of a few hours required for the establishment of a stable value of $\%$ long cells, changes in population density were relatively small, amounting to a factor of 2 or less, and the corresponding changes in \% long cells to be expected on the basis of the trend in Fig. 5 were comparable to their experimental errors. The control of the population density can therefore be regarded as adequate for these experiments.

Although on some occasions the dilution rate was deliberately altered from its steady-state value by as much as $\mathbf{5 0} \%$ for periods of one or two generation times, changes in the population of long cells were always small and could be attributed to changes in population density. Possible complications in the interpretation of length distribution as due to sudden changes in dilution rate (as discussed earlier) have therefore been neglected.

\section{Cell diameters}

The diameters of living cells were estimated visually to the nearest $0 \cdot 1 \mu$ using a $\times 90$ objective with phase contrast bright field illumination and with dark ground illumination. For 113 cells from one culture in the length range 2.5-30 $\mu$ the average diameters were $0.93 \pm 0.08 \mu$ with dark ground and $0.78 \pm 0.06 \mu$ with bright field. The difference between the two values can be ascribed to diffraction. When the measured diameters were divided into two equal groups about the median, the average diameters of the groups did not differ significantly.

\section{Search for evidence of selection}

The observations described about the dependence of average length upon temperature, population density and radiation could be explained in terms of phenotypic variation or of selection amongst clones of different average length. Experiments designed to distinguish between these two hypotheses showed that changes in the proportion of long cells due to changes in population density were reversible, whilst the proportions of long cells in cultures derived from single cells and from stock slopes did not differ significantly. An attempt to isolate a long-cell variant from a dilute culture at $37^{\circ}$ was not successful, the progeny of single cells after $\mathbf{3 0}$ generation times showing no significant differences in average length despite the widely differing lengths of the parent cells. 


\section{DISCUSSION}

Since we dealt exclusively with growing cultures it was not possible to determine whether the observed changes in length were due to selection or adaptation. Our failure to find evidence in our stock cultures for selection amongst two or more strains with very different tendencies to grow long forms has lead us to regard the observed changes as phenotypic responses to changing growth conditions. On this basis a change in average cell length of a population must be interpreted in terms of some change which tends to make all cells become longer or shorter. A change in average length implies a change in the length at which cells on the average divide; thus our experimental observations are broadly consistent with the simple model proposed above, if the average length at which cells divide is conditioned by the temperature, population density and the degree of gamma irradiation.

At $37^{\circ}$ a change in population density from $10^{6} / \mathrm{ml}$. to $2 \times 10^{8} / \mathrm{ml}$. produced no significant change in growth rate but a marked decrease in average cell length. A similar change in population density at $22^{\circ}$ also resulted in a decrease in cell length, but again the growth rate remained constant. Thus, a dependence of average cell size upon growth rate which Schaechter et al. (1958) found with Salmonella typhimurium certainly does not hold for Escherichia coli in minimal medium at population densities less than $2 \times 10^{8} / \mathrm{ml}$. On the other hand, when the results of Schaechter et al. (1958) for $S$. typhimurium are combined with similar ones for the dry weight of Aerobacter aerogenes and Bacillus megaterium (Herbert, 1959) and our own for E. coli, one finds that in all cases the forms of the dependence of size upon growth rate are almost identical in the region of suboptimal growth rates. We interpret this as indicating that the relation found by Schaechter et al. (1958) applies only when there is limitation of growth rate by partial exhaustion of the medium or limitation by accumulation of toxic substances.

The dependence of the \% long cells upon population density found in the present experiments suggests that the bacteria alter their growth medium in such a way as to inhibit the formation of long cells. This dependence is consistent with the hypothesis that bacteria remove an inhibitor present in the minimal medium or with the alternative hypothesis that they excrete a metabolite into it. Since gamma radiation also affects the $\%$ long cells, particularly at $\mathbf{2 2}^{\circ}$, the hypothetical inhibitor or metabolite may be a compound associated with a radiosensitive metabolic sequence.

We wish to thank Mr R. J. C. Hudson for the detailed design and construction of much of the apparatus.

\section{REFERENCES}

Alper, T. (1957). Observations on bacterial growth and morphology shortly after irradiation and some remarks on the oxygen effect. In Advances in Radiobiology, p. 90. Edinburgh and London: Oliver and Boyd.

Bryson, V. (1959). Applications of continuous culture to microbial selection. In Recent Progress in Microbiology, p. 371. Oxford: Blackwell's Scientific Publications.

DeErING, R. A. (1958). Studies on division inhibition and filament formation of Escherichia coli by ultraviolet light. J. Bact. 76, 123.

Fleming, A., Voureka, A., Kramer, I. R. H. \& Hughes, W. H. (1950). The morphology and motility of Proteus vulgaris and other organisms cultured in the presence of penicillin. J. gen. Microbiol. 4, 275. 
Henrici, A. T. (1928). Morphologic Variation and the Rate of Growth of Bacteria. London: Baillière, Tindall and Cox.

Herbert, D. (1959). Some principles of continuous culture. In Recent Progress in Microbiology, p. 381. Oxford: Blackwell's Scientific Publications.

Hinshelwood, C. N. \& Lodge, R. M. (1944). A physico-chemical study of some induced changes in the morphology of Bacterium lactis aerogenes. A theory of the balance of adaptive variation of certain enzyme processes in bacteria. Proc. Roy. Soc. B, 132, 47 .

Hughes, W. H. (1953). The origin of the L-form variants in anaerobic cultures of Bacterium coli. J. gen. Microbiol. 8, 307.

Lea, D. E., Haines, R. B. \& Coulson, C. A. (1937). The action of radiations on bacteria. III. Gamma rays on growing and non-proliferating bacteria. Proc. Roy. Soc. B, 123, 1.

LEDERBERG, J. (1950). Isolation and characterization of biochemical mutants of bacteria. Meth. med. Res. 3, 5.

Novick, A. \& Szilard, L. (1950). Experiments with the chemostat on spontaneous mutations in bacteria. Proc. nat. Acad. Sci., Wash. 36, 708.

Powell, E. O. (1955). Some features of the generation times of individual bacteria. Biometrika, 42, 16.

Powell, E. O. (1956). Growth rate and generation time of bacteria with special reference to continuous culture. J. gen. Microbiol. 15, 492 .

Powell, E. O. (1958). An outline of the pattern of bacterial generation times. J. gen. Microbiol. 18, 382.

Schaechter, M., MaAløe, O. \& KJeldgaard, N. O. (1958). Dependency on medium and temperature of cell size and chemical composition during balanced growth of Salmonella typhimurium. J. gen. Microbiol. 19, 592.

Spray, G. H. \& Lodge, R. M. (1943). The effects of resorcinol and of $m$-cresol on the growth of Bact. lactis aerogenes. Trans. Faraday Soc. 39, 424.

WITKIN, E. M. (1947). Genetics of resistance to radiation in Escherichia coli. Genetics, 32, 221. 